\title{
The Study of Simulation Metamodel Based on Bayesian Network and Its Application in Equipment Battlefield Damage Simulation
}

\author{
Hai-Kuan WANG ${ }^{1, a^{*}}$, Quan SHI ${ }^{1}$, Guang-Yan WANG ${ }^{1}$ and Hong-Yu GE ${ }^{1}$ \\ 11. Department of Equipment Command \& Management, Mechanical Engineering College, \\ Shijiazhuang, 050003, China \\ awww.whk_01@163.com
}

Keywords: Equipment, Simulation, Original model, Metamodel, Bayesian network, Battlefield damage.

\begin{abstract}
The construction method of simulation metamodel based on Bayesian network is studied for the equipment battlefield damage simulation system. The mapping relation between input parameters and output parameters of simulation model is described from the position of condition probability, the feasibility of construction of metamodel based on Bayesian network is studied, the merits of metamodel based on Bayesian network are analyzed. The key problems are studied in the process of construction of metamodel based on Bayesian network, including the ensuring of metamodel parameters, the translation from original model parameters to Bayesian network nodes, the calculation of joining intensity, and the construction of derived metamodel. Aiming at equipment battlefield damage fast location without complete information, the construction method of metamodel based on Bayesian network is studied based on the K2 method. The metamodel based on Bayesian network is constructed for one type of antiaircraft artillery.
\end{abstract}

\section{Introduction}

Metamodel is a simple alternative model simulation model, by entering the original model - an output sequence obtained by fitting to get the approximate mathematical model . Because the simulation metamodel does not care about the inner work of the original model, so it has simple structure, can reduce the complexity of the original model, significantly reduce the computation overhead, so the simulation metamodel approach has become a hot topic in the emerging field of the current system simulation ${ }^{[1]}$.

The common simulation modeling method including polynomial regression analysis, multivariate adaptive regression splines, radial basis function, neural networks ${ }^{[2,3]}$. This paper focuses on the simulation meta-model based on Bayesian network construction method, and the method is used in Battlefield Damage Simulation modeling process.

\section{Bayesian network simulation metamodel Fundamentals}

\section{Bayesian network structure principle}

Given a joint probability distribution $P\left(X_{1}, X_{2}, \ldots, X_{n}\right)$ and a sort of variables d, put $X_{1}$ as a root, and to give $X_{1}$ a priori probability distribution $P\left(X_{1}\right)$, Then expressed $X_{2}$ by a node, if $X_{2}$ and $X_{1}$ are related, From $X_{1}$ to $X_{2}$ establish a link, and represented connection strength by $P\left(X_{2} \mid X_{1}\right)$. If $X_{2}$ and $X_{1}$ is independent, then give $X_{2}$ a priori probability distribution $P\left(X_{2}\right)$. In the first stage $i$, from $X_{i}$ parent node congregation $\prod_{X_{i}}\left(\prod_{X_{i}} \subseteq\left\{X_{1}, X_{2}, \ldots, X_{i-1}\right\}\right)$, draw a set of directional line 
coupling $X_{i}$, And quantified conditional probabilities by $P\left(X_{i} \mid \Pi_{X_{i}}\right)$, can get a directed acyclic graph, can be used to represent a number of independent relations embodied of $P\left(X_{1}, X_{2}, \ldots, X_{n}\right)$, this figure is known as Bayesian networks ${ }^{[4]}$.

In turn, $P\left(X_{i} \mid \Pi_{X_{i}}\right)$ contains all of the information which is necessary for the original distribution function reconstruction. Under the sort $d$, has the following relationship, link rules:

$$
P\left(X_{1}, X_{2}, \ldots, X_{n}\right)=\prod_{i} P\left(X_{i} \mid \prod_{X_{i}}\right)
$$

Once the network is established, it can be used to represent a general knowledge of the field, and can be used for reasoning, Bayesian networks are not just static store some factual knowledge, but also as a computational strategy for these knowledge reasoning. In the case of the evidence $e$, the conditional probability of the event $\mathrm{X}$ is reflected the possibility of an event occurring in certain circumstances .

When obtain the evidence $e$, the network beliefs are updated, obtaining $\operatorname{Bel}(X)=P(X \mid e)$ specific values of faith.

\section{The conditional probability characteristic of analysis the simulation model input parameters and output parameters}

The simulation model reflects the mapping relationship between the input parameters $I_{1}, I_{2}, \ldots, I_{u}$ and the output parameters $O_{1}, O_{2}, \ldots, O_{v}$, so $\left(O_{1}, O_{2}, \ldots, O_{u}\right)=f\left(I_{1}, I_{2}, \ldots, I_{v}\right)$ For this mapping can be re- interpreted from the perspective of the conditional probability, translating $f$ into a conditional probability $P\left(O_{1}, O_{2}, \ldots, O_{u} \mid I_{1}, I_{2}, \ldots, I_{v}\right)$, it actually reflects the mapping between the $f$ consistent with the simulation model, which can describe the mapping between simulation model input parameters and output parameters by the Bayesian network. Bayesian network update the entire network based on the observed information, which is given in the form of posterior probability $\operatorname{Bel}(X)=P(X \mid e)$.From the view of the simulation metamodel input parameters and output parameters, the Bayesian posterior probability can be regarded as a kind of network mapping between the input parameters and output parameters, considering evidence $e$ as input parameters of the element model simulation, The posterior probability of the other nodes $X$ in the network may be regarded as the output parameter simulation metamodel $\left(O_{1}, O_{2}, \ldots, O_{u}\right)=f\left(I_{1}, I_{2}, \ldots, I_{v}\right) \rightarrow$ $P\left(O_{1}, O_{2}, \ldots, O_{u} \mid I_{1}, I_{2}, \ldots, I_{v}\right)$.

Because there are certain intrinsic link between the input parameters and output parameters of the simulation model, whereby certain Bayesian network can be constructed in accordance with the rules of the link, available to solve critical data $P\left(O_{1}, O_{2}, \ldots, O_{u} \mid I_{1}, I_{2}, \ldots, I_{v}\right)$, the joint probability distribution of all parameters: $P\left(O_{1}, O_{2}, \ldots, O_{u}, I_{1}, I_{2}, \ldots, I_{v}\right)$, enabling the use of Bayesian network meta-model instead of the original simulation model for simulation purposes.

\section{Advantages of Bayesian network simulation metamodel}

The original model into the corresponding Bayesian network meta-model simulation, using Bayesian belief network to update the mapping between the principle instead of the original model, relative to other simulation modeling method has the following advantages :

(1)Flexible simulation model to reflect the multi-layer mapping between the input parameters and output parameters between

Conventional element modeling methods typically reflect single parameter mapping between input parameters and output parameters between the fixed structure or multi- parameter mapping. The Bayesian network meta-model simulation is based on the statistical dependence relationship between parameters constructed, expressed as a parameter with the input and output parameters between the 
input - output sequence changes constantly self-adjusting, flexible multi- mappings, thereby enhancing the ability to fit the data element model simulation, while enhancing the intelligibility of simulation metamodel to better explain the relationship between the input parameters are passed to the information output parameters.

(2)Flexibility to adjust the format of the simulation model input parameters

Conventional meta-model input parameters are usually fixed format, mainly in two aspects: 1, in the form of fixed parameters, or the use of deterministic parameters, or the use of probabilistic parameters ; 2, the fixed number of parameters, namely the need for each parameter are given a certain values, does not allow parameters vacancies. The input parameters of Bayesian network simulation metamodel can be flexibly adjusted in two ways: 1, you can enter parameter uncertainty, $I_{i}$ take a state probability $z_{k}$ is 1 , the probability of taking the other states is zero. It can also enter a probabilistic arguments $P\left(I_{i}=z_{k}\right)=p_{k} \quad\left(0<p_{k}<1\right) ; 2$, If the parameter $I_{i}$ is difficult to determine, without their assignment, marginalization method by $P\left(O_{1}, O_{2}, \ldots, O_{n} \mid I_{1}, I_{2}, \ldots, I_{m}\right)$ converted to $P\left(O_{1}, O_{2}, \ldots, O_{n} \mid I_{1}, \ldots, I_{i-1}, I_{i+1}, \ldots, I_{m}\right)$.

(3)Build flexible forms of simulation metamodel.

Bayesian network simulation element model consists of two parts, one is the basic element model, which is consistent with the original model function, reflecting the initial simulation metamodel mapping between the original model; the second is derived from meta-model that, according to research needs in basic extracting the corresponding input parameters and output parameters based on the meta-model, constitute a particular derivative models. Selected input parameters and output parameters are different, derived form metamodel is different.

\section{The key technology to build a Bayesian network simulation metamodel}

Node and link strength are the two basic elements of Bayesian networks, and construct a Bayesian network model is the core element derived Bayesian network simulation metamodel, this section focus on these key issues, the researchers constructed a Bayesian network some of the key technical element model simulation .

\section{Bayesian network meta-model to determine the parameters of the simulation}

According to research, firstly, it needs to determine the parameters of Bayesian networks. According to the relationship between the parameters of the original model, the parameters can be divided into three categories, one input parameter, which is the starting point for analysis of the problem ; the second is an output parameter, which is the ultimate objective analysis of the problem ; the third is transitional parameters, that in order to facilitate the establishment and reflect the mapping between input parameters and output parameters between some intermediate transitional parameters within the original model introduced in order to more clearly describe the input parameters to the output parameters of the transformation process . Obviously, the input and output parameters for meta-modeling staff is fully visible, and visibility problems transitional parameters is more complex . Visible for transitional original model, Bayesian network parameters to determine more convenient, you can determine the relationship between the internal operations of the original model of Bayesian network parameters ( including input parameters, output parameters, and transition parameters ) based on ; for transition parameters invisible original model to determine the parameters of Bayesian networks is more difficult, usually only to determine the input and output parameters of the original model, but it can not determine the parameters of the transition between them . For transitional original model parameters are not visible, because of the lack of complete information about the parameter, the difficulty of establishing a Bayesian network simulation metamodel larger, the accuracy is relatively reduced. Bayesian network element determination process simulation model parameters is shown in Figure 1. 


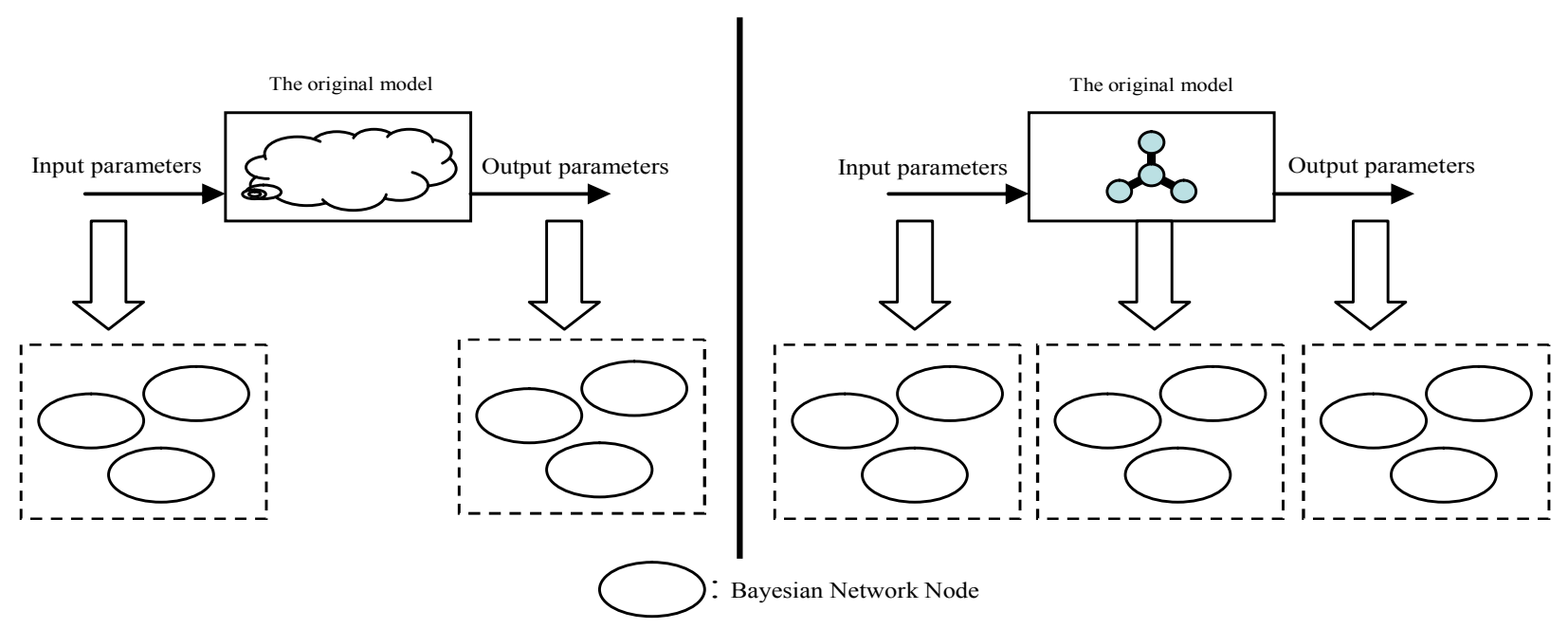

(a) Transitional parameters are not visible

(b) Transitional parameters are visible

Fig. 1 Bayesian network meta-model parameter determination process simulation schematic

\section{Bayesian Network node from the conversion of the original model parameters}

If the original model parameters are discrete values, the conversion process is very simple, for each discrete parameters, namely the design of a node, based on the value of discrete parameters of the situation, given the appropriate status for the node can be.

The existence of parameter discrete $X_{i}$ has a number of $s$, they are $\left(x_{1}, x_{2}, \ldots\right)$, Respectively corresponding to $\left(x_{1}, x_{2}, \ldots\right)$; If the original model parameters is continuous quantity, it need the original model based on the accuracy requirements, the continuous parameters into a finite number of discrete values, the same transformation parameters to the node can be achieved, the conversion process as shown in the following formula.

$$
X=\left\{\begin{array}{l}
z_{1}, X_{0} \leq X<X_{1} \\
z_{2}, X_{1} \leq X<X_{2} \\
\cdots \\
z_{s}, X_{s-1} \leq X<X_{s}
\end{array}\right.
$$

Continuous parameters into a finite number of discrete node status is a complex task, discrete node status, the more the higher model accuracy, but will lead to the Bayesian network structure is too complicated ; Fewer discrete state model accuracy will be reduced, but will make the Bayesian network structure is simplified. Therefore, it should be between network structure and calculation accuracy tradeoff in order to meet the accuracy requirements of the premise, the network structure has been simplified to a great extent .

\section{The mapping of the original model Transforming to the intensity of Bayesian network node links}

For the original model, as reflected in the mapping e, needs to be translated to the coupling strength between nodes in the Bayesian network.

If the Bayesian network structure is known, you can enter the original model - the output sequence, based on the parameters by maximum likelihood estimation method, respectively, to determine the coupling strength between the nodes Considering a composition of $n$ variables $\mathrm{X}=\left\{X_{1}, X_{2}, \ldots, X_{n}\right\}$ Bayesian network $\mathcal{N}$, located where the nodes $X_{i}$ total value of $r_{i}$, the parent node $\pi\left(X_{i}\right)$ has total value of $q_{i}$ combinations. 
If $X_{i}$ has no parent node, $q_{i}=1$. And coupling strength between the Bayesian network nodes: $\theta_{i j k}=P\left(X_{i}=k \mid \pi\left(X_{i}\right)=j\right)$. For a fixed $i, j$ and $k$ ranges $1 \sim q_{i}$ and $1 \sim r_{i}$, assuming $\mathcal{D}=\left(\mathrm{D}_{1}, \mathrm{D}_{2}, \ldots, \mathrm{D}_{m}\right)$ as a complete of data for i.i.d. about $\mathcal{N}$, and $\mathrm{D}_{l}$ define the characteristics of the sample as a function of $\chi\left(i, j, k ; \mathrm{D}_{l}\right)$ :

$$
\chi\left(i, j, k ; \mathrm{D}_{l}\right)=\left\{\begin{array}{l}
1, \text { if } X_{i}=k, \text { and } \pi\left(X_{i}\right)=j \\
0, \text { if not }
\end{array}\right.
$$

And set: $m_{i j k}=\sum_{l=1}^{m} \chi\left(i, j, k ; \mathrm{D}_{l}\right)$, then the maximum likelihood estimate of $\theta_{i j k}$ :

$\hat{\theta}_{i j k}=\left\{\begin{array}{l}\frac{m_{i j k}}{\sum_{k=1}^{r_{i}} m_{i j k}}, \text { if } \sum_{k=1}^{r_{i}} m_{i j k}>0 \\ \frac{1}{r_{i}}, \text { if } n o t\end{array}\right.$

The intuitive meaning of maximum likelihood estimation method is:

$$
\hat{\theta}_{i j k}=\frac{\text { the number of samples in } \mathcal{D} \text { which satisfy } X_{i}=k \text { and } \pi\left(X_{i}\right)=j}{\text { the number of samples in } \mathcal{D} \text { which satisfy } \pi\left(X_{i}\right)=j}
$$

If the Bayesian network structure is unknown, it need a structured learning approach, in obtaining the structure of the underlying network topology on the best, and then estimate the corresponding coupling strength $\theta_{i j k}$, in Section 4 of this article based on Bayesian network structure K2 algorithm learning methods.

\section{Construction of the Bayesian network simulation metamodel derived element model}

By determining Bayesian network parameters and transformation coupling strength, It is available to get basic element models with the original model, reflects the basic the mapping of the original model between input parameters and the output parameters. The basic element model has a strong scalability, extracting different input parameters and output parameters in the basic element model, constitute different derivatives metamodel, in order to meet the different requirements of the simulation system. Derivative element model building process shown in Figure 2, Corresponding basic element model based on the original model, namely Bayesian networks $\{A, B, C, D, E, F\}$, accordingly can generate several different derivative element model reflects the input parameters and output parameters of the mappings ${ }^{\mathcal{N}}{ }_{i}$, for example $P(F \mid A, B)$, It reflects the mapping of $F=f(A, B)$; or $P(D \mid C, E)$, It reflects the mapping of $D=f(C, E)$. 


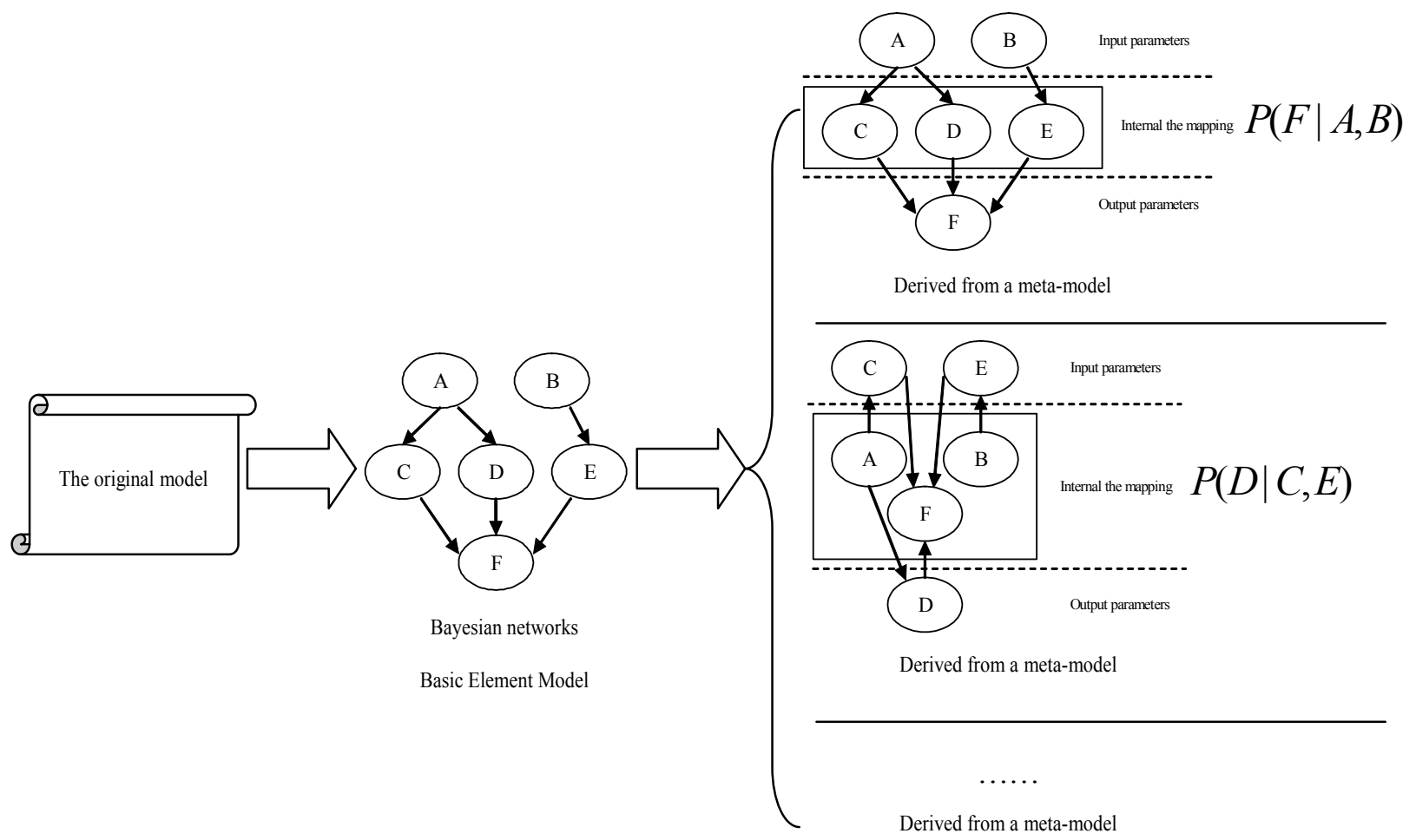

Fig. 2 derived Bayesian network meta-model Construction of schematic

\section{Element model based on Bayesian network simulation algorithm K2 construction method}

Bayesian network meta-model simulation study in this section refers to the basic element model, its original model simulation focused on construction methods and equipment for battle damage , injury status mutual focus on equipment components that occur under projectile fragments between Field Contact .

\section{The causality of equipment Parts battlefield damage}

Fighting equipment damage occurring in the battlefield environment is a very typical common cause damage, leading to the threat source is usually the role of fighting in damage to equipment and equipment as a whole, is not limited to certain parts of the equipment damage between the various components are not independent of each other .

It Has been developed Battlefield Damage Simulation System, using the system of basic data and equipment available in certain parts of the operational environment of each battlefield injuries occur, the damage correlation between the paper focuses on data and equipment parts, in order to solve Battlefield Damage quickly locate the problem. Because in the real battlefield environment, the evaluators are often difficult to obtain comprehensive information about all the damage the equipment ( such as equipment, all of the lesion, degree of injury and other injury site ), only part of the damage information to obtain equipment, which is mainly composed of equipment structural characteristics of the decision[5], such as certain parts of smaller size or located inside the equipment, it is difficult to directly observe damage condition[6] . In this paper, Battlefield Damage Simulation System ( original model ), based on data collected a lot of equipment damage sequence parts, thus construct the corresponding Bayesian network meta-model simulation to solve the conduct Battlefield Damage positioned under incomplete information issue.

Equipment Battlefield Damage Simulation original model input parameters for injury status of a component, the output parameters for damage to other parts of the state, we use a Bayesian network meta-model instead of the original simulation model to reproduce the original model, as reflected in the mapping relationship. Emphasis on the mapping of the method and equipment damage parameters 
between the various components, which reflect a causal relationship mapping between components injury. This causal relationship can be understood: the equipment is damaged under certain external energy action will occur, And this external energy acting on the equipment as a whole, and in accordance with certain parts ordering ( energy pathway ) in order to pass backwards, resulting in energy transfer can be considered as the energy pathways of the back-end components to injury from its front end parts, So as to establish a causal relationship between certain parts of each injury, which is located in the front part of energy pathway is the cause of the back-end component damage occurs , the front-end component damage does not occur, the back-end components and certainly will not happen damage. For fragmentation damage energy for the energy transfer pathways for individual parts of sorting through the fragments shown in Figure 3.

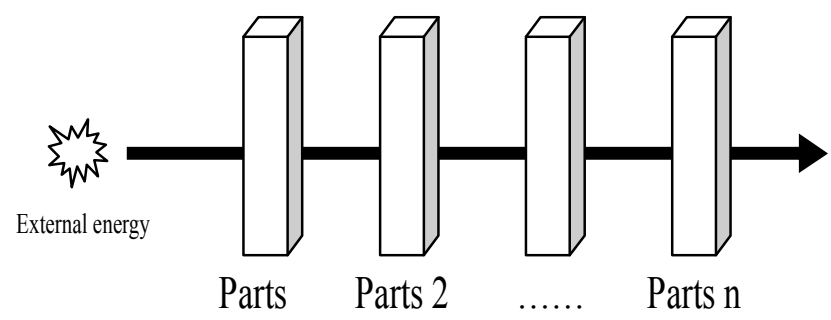

Fig. 3 Fragments energy pathway schematic

However, due to the complex structure of the equipment, the energy transfer pathway threats difficult to accurately determine, thus resulting in a Bayesian network topology is also difficult to determine, it can be considered as transitional invisible original model parameters, to solve this problem, in this paper, K2 algorithm for Bayesian network topology between the parts and equipment damage.

\section{K2 algorithm for Bayesian network structure learning}

Structure learning is generally divided into two steps, namely, model selection and model optimization, model selection criteria to solve any kind of problem used to judge the merits of different models for the structure of the model to optimize the optimal model structure should find out.

\section{Bayesian network optimization criterion score}

Assuming $\zeta$ as a Bayesian network structure node with $X_{1}, X_{2}, \ldots, X_{n}, \zeta$ can use a scoring function to measure, This paper use $\mathrm{CH}$ scoring criteria. Assuming prior distribution parameters obey product Dirichlet distribution: $\prod_{i=1}^{n} \prod_{j=1}^{q_{i}} \prod_{k=1}^{r_{i}} \theta_{i j k}^{\alpha_{i j k}-1}$,Assuming $\mathcal{D}=\left(\mathrm{D}_{1}, \mathrm{D}_{2}, \ldots, \mathrm{D}_{m}\right)$ is a complete set of data on the variablesi.i.d., the optimal parameters for the log-likelihood function is:

$$
\begin{aligned}
& l(\zeta \mid \vec{D})= \\
& \sum_{i=1}^{n} \sum_{j=1}^{q_{i}}\left[\log \frac{\Gamma\left(\alpha_{i j^{*}}\right)}{\Gamma\left(\alpha_{i j^{*}}+m_{i j^{*}}\right)}+\sum_{k=1}^{r_{i}} \log \frac{\Gamma\left(\alpha_{i j k}+m_{i j k}\right)}{\Gamma\left(\alpha_{i j k}\right)}\right]
\end{aligned}
$$

The amount of formula (1) given by the right side is called the structure $\mathrm{CH}$ Rating $m_{i j^{*}}=\sum_{k=1}^{r_{i}} m_{i j k}, \alpha_{i j^{*}}=\sum_{k=1}^{r_{i}} \alpha_{i j k}$. If we assume that the structure of the meaning of the prior distribution is uniform, then select the model is equivalent to a Bayesian score with $\mathrm{CH}$ selection model. 
$\mathrm{CH}$ can be considered as a sum of scores, one for each variable. Using $<X_{i}, \pi\left(X_{i}\right)>$ note the variable $X_{i}$, parent node $\pi\left(X_{i}\right)$ and the local edge structure is formed. Equation (1) with $X_{i}$ the corresponding one that depends on the local structure $<X_{i}, \pi\left(X_{i}\right)>$. So

$$
\begin{aligned}
& \mathrm{CH}\left(<X_{i}, \pi\left(X_{i}\right) \mid \vec{D}>\right)= \\
& \sum_{j=1}^{q_{i}}\left[\log \frac{\Gamma\left(\alpha_{i j^{*}}\right)}{\Gamma\left(\alpha_{i j^{*}}+m_{i j^{*}}\right)}+\sum_{k=1}^{r_{i}} \log \frac{\Gamma\left(\alpha_{i j k}+m_{i j k}\right)}{\Gamma\left(\alpha_{i j k}\right)}\right]
\end{aligned}
$$

$\mathrm{CH}\left(<X_{i}, \pi\left(X_{i}\right)>\mid \vec{D}\right)$ is the score of family $\mathrm{CH}$ for $X_{i}$, Use family $\mathrm{CH}$ score, formula (1) can be rewritten as:

$$
\mathrm{CH}(\zeta \mid \vec{D})=\sum_{i=1}^{n} \mathrm{CH}\left(<X_{i}, \pi\left(X_{i}\right)>\mid \vec{D}\right)
$$

Equation (2) is dependent on the right in $\pi\left(X_{i}\right)$ network structure $\zeta$, In the sense of the formula described, $\mathrm{CH}$ score is decomposable, it is broken down into each variable family $\mathrm{CH}$ score sum. In the structure of the learning process, every step of the current model structure $\zeta$ slightly modified, get another structure $\zeta^{\prime}$, then calculate and compare the two scores. Usually there is little difference between $\zeta$ and $\zeta^{\prime}$, the parent node only one or two variables change. If the scoring function is decomposable, then the difference between $\zeta$ and $\zeta^{\prime}$ is one or two variables score change, it is easy to calculate. Assuming only difference between $\zeta$ and $\zeta^{\prime}$ is the parent node of a variable changes, from $\pi\left(X_{i}\right)$ become $\pi^{\prime}\left(X_{i}\right)$, then:

$$
\begin{aligned}
& \mathrm{CH}\left(\zeta^{\prime} \mid \vec{D}\right)-\mathrm{CH}(\zeta \mid \vec{D})= \\
& \mathrm{CH}\left(<X_{i}, \pi^{\prime}\left(X_{i}\right)>\mid \vec{D}\right)-\mathrm{CH}\left(<X_{i}, \pi\left(X_{i}\right)>\mid \vec{D}\right)
\end{aligned}
$$

\section{K2 algorithm for structure learning}

$\mathrm{K} 2$ algorithm is one of the Bayesian network structure learning algorithm earliest. Assuming $\vec{D}$ as a complete set of data on $X_{1}, X_{2}, \ldots, X_{n}$ of i.i.d. The purpose of $\mathrm{K} 2$ algorithm is to find high scoring model $\mathrm{CH}$. Computational complexity considerations, $\mathrm{K} 2$ is not to find out the highest score $\mathrm{CH}$ model, but under certain conditions, to find the best model. There are two conditions employed, they are greatly reduced so that the search space. K2 algorithm uses a variable sort $\rho$ and a positive integer $u$ to limit the search space It must seek to satisfy the following two conditions are optimal model $\zeta$ :

(1) The number of any parent node from $\zeta$ does not exceed $u$;

(2) $\rho$ is a topological sort of $\zeta$.

To simplify the calculation of model scores, $\mathrm{K} 2$ prior distribution is assumed that all parameters are evenly distributed, that means $\alpha_{i j k}$ and $\alpha_{i j^{*}}$ both equal 1 .

$\mathrm{K} 2$ algorithm is the starting point that contains all the nodes, but not infinity edge map. In the search process, $\mathrm{K} 2$ sequentially investigates one by one form $\rho$ variables to determine its parent node, and then add the appropriate side. for $X_{j}$, Assuming $\mathrm{K} 2$ has found some of its parent node $X_{j}$. If $\left|\pi_{j}\right|<u$, That is the number $\left|\pi_{j}\right|$ of parent node $X_{j}$ has not reached $u$, then it is necessary to continue to look for the parent node. In practice, the first consideration of those ahead of $X_{j}$ in $\rho$, but it is not the 
variable $\mathrm{q}$ parent node, choose $X_{i}$ from them, it makes the new family of $\mathrm{CH}$ score $V_{\text {new }}=\mathrm{CH}\left(<X_{j}, \pi_{j} \cup\left\{X_{i}\right\}>\mid \vec{D}\right)$ reached the maximum; and compare $V_{\text {new }}$ and $\mathrm{CH}$ score $V_{\text {old }}=\mathrm{CH}\left(<X_{j}, \pi_{j}>\mid \vec{D}\right)$; If $V_{\text {new }}>V_{\text {old }}$, then put $X_{i}$ as the parent node of $X_{j}$; otherwise, stop looking for parent of $X_{j}$.

\section{Variable ordering statistical method of determining $\rho$}

Sort variable $\rho$ for Bayesian network structure plays a key regulatory role, the variables can be regarded as higher-ranking cause of event sequencing rearward variables. According Equipment Battlefield Damage occurs correlation characteristics can determine $\rho$ according to the energy pathway, but the energy transfer pathway is a complex task to determine, however, to determine the energy transfer pathway is a complex task, in this paper, the energy transfer process will occur in accordance with the attenuation characteristics, using statistical methods to determine a more concise variable ordering $\rho$, the average value of the received energy component is determined.

Node energy transfer process performed inside the equipment, will gradually decay occurs, the energy transfer pathway located at the distal end of the received energy is large, while at the rear end of the energy received by a node is small, the energy transfer pathway in accordance with the energy is gradually attenuated, as shown in Figure 4. According to the characteristics of energy transfer, respectively statistics of the received energy component size, the greater the energy received by a node, its position in the ordering of the front, variable ordering is determined based on the size of the received energy.

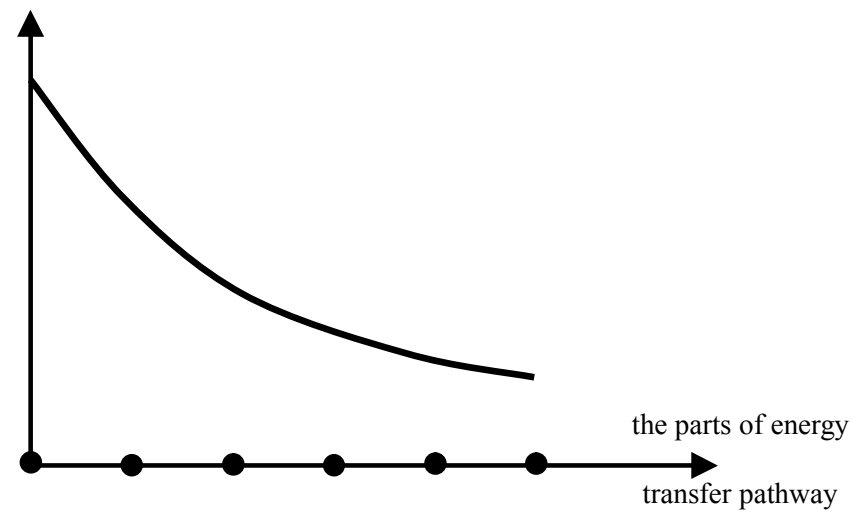

Fig. 4 schematic energy attenuation

For Fragment Penetrating energy equipment exists a set of $n$ parts, equipment set up around a burst point $p$, for $n$, exist $p$ group fragments hit values $e_{i, j}(i$ represents the number, $j$ represents fried point number), $e_{i}$ is the average received power of each component is the average number of fragments that hit parts description available, $e_{i}=\frac{\sum_{j=1}^{p} e_{i, j}}{p}$,it can be determined according to the size of the variable $e_{i}$ ordering sort $\rho$

\section{The study of Bayesian network simulation metamodel for a kind of antiaircraft}

In this paper, the problem quickly locate equipment battlefield injuries studied antiaircraft battle damage simulation Bayesian network metamodel. The simulation metamodel for Battlefield Damage Simulation System is the original model, to research the relationship between the main focus of equipment damage components. 


\section{Antiaircraft parts for the Bayesian network simulation metamodel built}

Focuses on the relationship between the components are damaged : the Antiaircraft barrel, the level of machine , Defensive Shield, brackets, sights , Antiaircraft -bed cross beam Antiaircraft legs, wheels, cradle, direction . These components are relatively large component shape, but also prone to damage in parts of the projectile fragments Field.

\section{Damaged Equipment basic data collection}

In order to explore the relationship between the parts and equipment damage, you need to get a lot of equipment parts battle damage data. To do this, set up a large number of point of impact at the close of the equipment, spare parts and equipment to collect the average number of hits shrapnel. According to the average number of hits shrapnel would damage state map into four parts : Misses shrapnel ( state 1 ), hit a shrapnel ( state 2 ), hit two shrapnel ( state 3 ), hit three or more shrapnel ( state 4 ). Were set up equipment close at 110 burst point, the group won the 110 battle damage data equipment parts.

\section{Determining Equipment parts order}

According to the principle of $\mathrm{K} 2$, sort front parts are sorted by cause injury occurred after the parts, so when building Bayesian network equipment battle damage simulation meta-model, according to the number of components to determine the average variable ordering shrapnel hits, Average shrapnel hit a few more, the parts ordering more front . Accordingly determine the sort of parts: Wheels (C1), leg (C2), Defensive Shield (C3), sight (C4), cross beam bed (C5), barrel (C6), the bracket (C7), the direction of the machine (C8), shake frame (C9), the level of machine (C10), shrapnel hit several parts of the average shown in Figure 5.

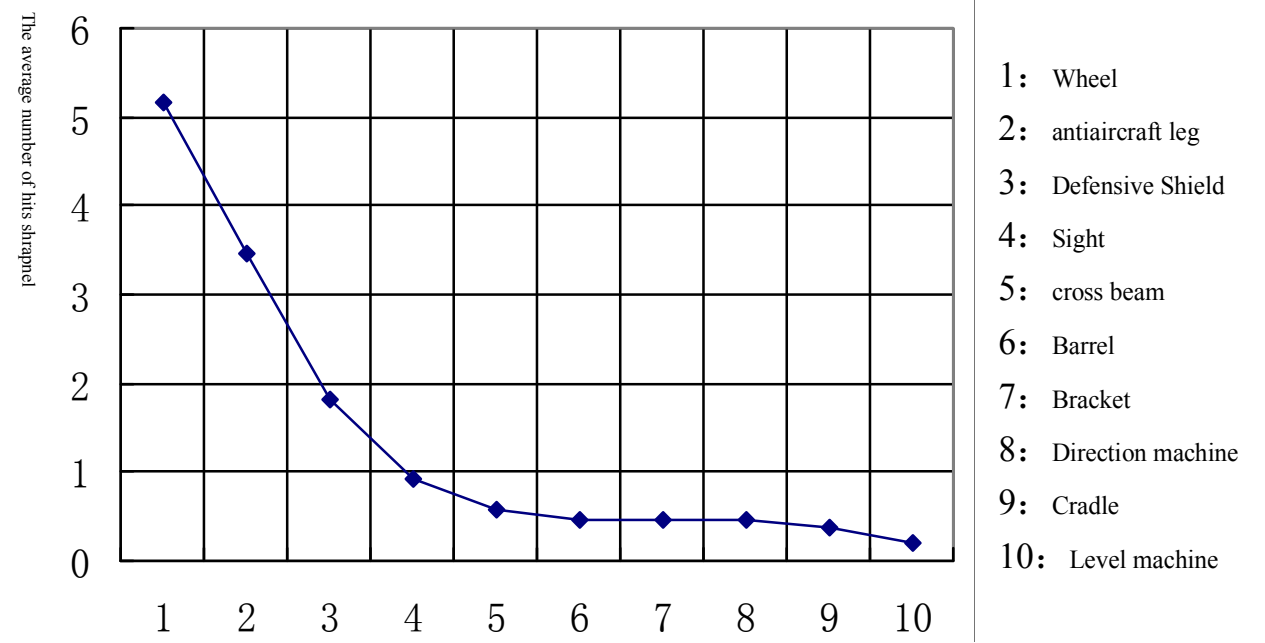

Fig. 5 The average number shrapnel hits for parts

Collectively this sort reflects the size of the space occupied by ordering parts. Focuses on the average number of shrapnel hit the most with the least two parts, wheels and height machine. Due to the location of detonating ammunition on the ground, so the spatial position of these two components, the wheel easier shrapnel hit, and position relative to the level of the machine is located inside the equipment, not easy to be shrapnel hit. Will be seen as a lead level of machine wheel damage occurs is reasonable cause event that wheel damage does not occur, the level of the machine is difficult injury.

\section{Bayesian network structure learning}

According to the above equipment parts ordering, using the $\mathrm{K} 2$ algorithm corresponding Bayesian network meta-model simulation, the basic element model, shown in Figure 6. 


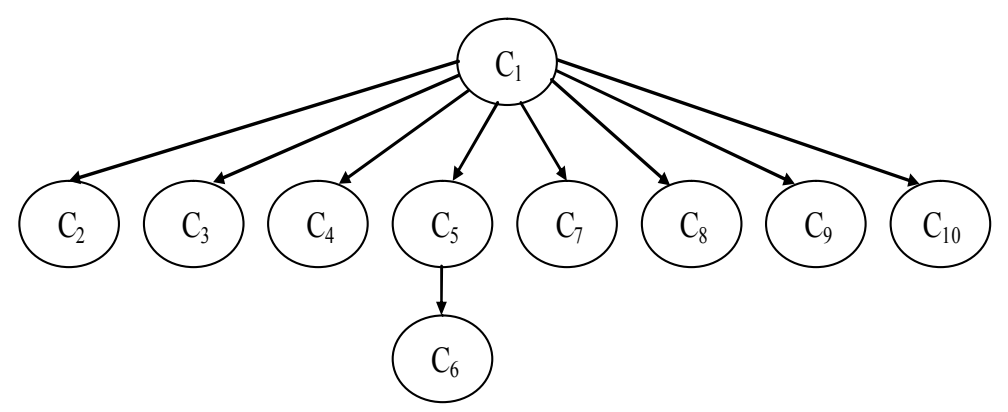

Fig. 6 Bayesian network topology basic metamodel

In the Bayesian network structure learning process, each network node respectively sorted to find the optimum position of the structure, thus increasing the overall network $\mathrm{CH}$ score in the learning process , $\mathrm{CH}$ trend value shown in Figure 7 .

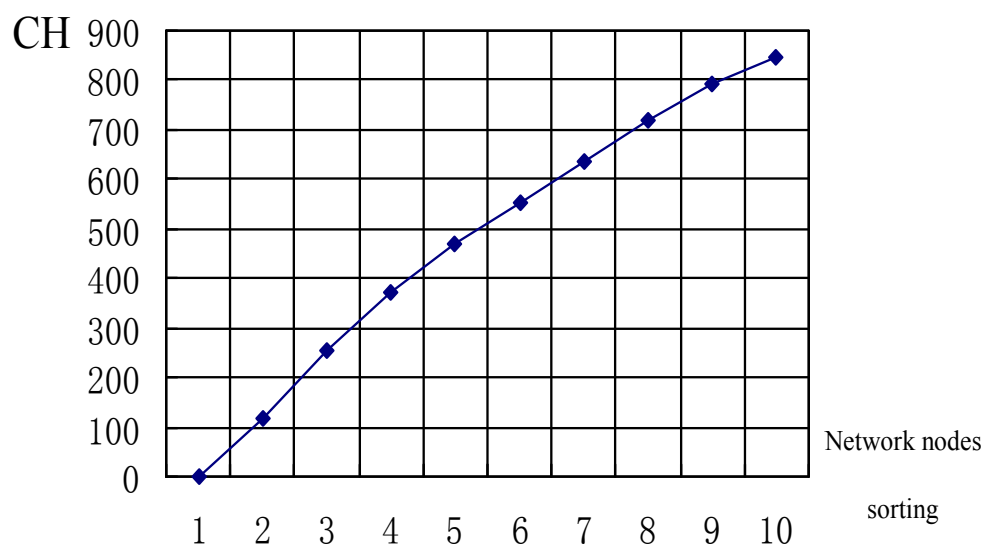

Fig. $7 \mathrm{CH}$ scores trend chart of structure the learning process

\section{Bayesian network simulation metamodel}

The basic element model reflects the basic causal link between the damage these components, the basic element model is now based on a number of derivative element model constructed to meet the different requirements quickly locate damage .

Wheel damage state analysis based on the cradle damage state. Cradle node is about fundamental element model (C9) as an input parameter model, the wheels node (C1) regarded as the output parameters of the model, the other node as an intermediate transition parameters, shown in Figure 8 (a). Output of the model are shown in Table 1, on the whole, the probability of occurrence of damage to the state 4 wheels is very high, in the proximity point case insensitive wheel damage state for cradle damage state changes.

Table 1 input parameters and output parameters derived model 1

\begin{tabular}{l|l|l}
\hline \multirow{2}{*}{$\begin{array}{c}\text { Input parameters } \\
\text { (Cradle damage state) }\end{array}$} & \multicolumn{2}{|c}{$\begin{array}{c}\text { Output parameters } \\
\text { (Wheel damage state probability }(\%))\end{array}$} \\
\hline \multirow{4}{*}{ state1 } & state1 & 1.61 \\
\cline { 2 - 3 } & state2 & 7.01 \\
\cline { 2 - 3 } & state3 & 8.63 \\
\cline { 2 - 3 } & state4 & 82.75 \\
\hline \multirow{4}{*}{ state2 } & state1 & 1.01 \\
\cline { 2 - 3 } & state2 & 7.62 \\
\cline { 2 - 3 } & state3 & 7.78 \\
\cline { 2 - 3 } & state4 & 83.58 \\
\hline \multirow{2}{*}{ state3 } & state1 & 1.17 \\
\cline { 2 - 3 } & state2 & 6.40 \\
\hline
\end{tabular}




\begin{tabular}{l|l|l}
\hline \multirow{4}{*}{ state4 } & state3 & 6.27 \\
\cline { 2 - 3 } & state4 & 86.15 \\
\hline \multirow{4}{*}{} & state1 & 0.54 \\
\cline { 2 - 3 } & state2 & 6.83 \\
\cline { 2 - 3 } & state3 & 6.41 \\
\cline { 2 - 3 } & state4 & 86.23 \\
\hline
\end{tabular}

(2) Wheel damage state analysis based on state Antiaircraft leg injury

Antiaircraft leg node upcoming basic element model (C2) as an input parameter model, the wheels node $(\mathrm{C} 1)$ regarded as the output parameters of the model, the other node as an intermediate transition parameters, shown in Figure 8 (b). Output of the model shown in Table 2 , on the whole, in the case of proximity points, wheel damage state artillery leg injuries for increased sensitivity to changes in the state, that is, with increasing state Antiaircraft leg injury, damage occurs state 4 wheels probability increases.

Table 2 derived model input parameters and output parameters 2

\begin{tabular}{l|l|l}
\hline $\begin{array}{c}\text { Input parameters(antiaircraft leg } \\
\text { injury status) }\end{array}$ & \multicolumn{2}{|c}{$\begin{array}{c}\text { Output parameters } \\
\text { (Wheel damage state probability }(\%)\end{array}$} \\
\hline \multirow{4}{*}{ state1 } & state 1 & 4.35 \\
\cline { 2 - 3 } & state2 & 17.39 \\
\cline { 2 - 3 } & state3 & 8.70 \\
\cline { 2 - 3 } & state4 & 69.56 \\
\hline \multirow{4}{*}{ state 2 } & state1 & 0 \\
\cline { 2 - 3 } & state2 & 8.70 \\
\cline { 2 - 3 } & state3 & 17.39 \\
\cline { 2 - 3 } & state4 & 73.91 \\
\hline \multirow{4}{*}{ state 3 } & state1 & 0 \\
\cline { 2 - 3 } & state2 & 0 \\
\cline { 2 - 3 } & state3 & 14.29 \\
\cline { 2 - 3 } & state4 & 85.71 \\
\hline \multirow{4}{*}{ state 4 } & state1 & 0 \\
\cline { 2 - 3 } & state2 & 4.00 \\
\cline { 2 - 3 } & state3 & 0 \\
\cline { 2 - 3 } & state4 & 96.00 \\
\hline
\end{tabular}



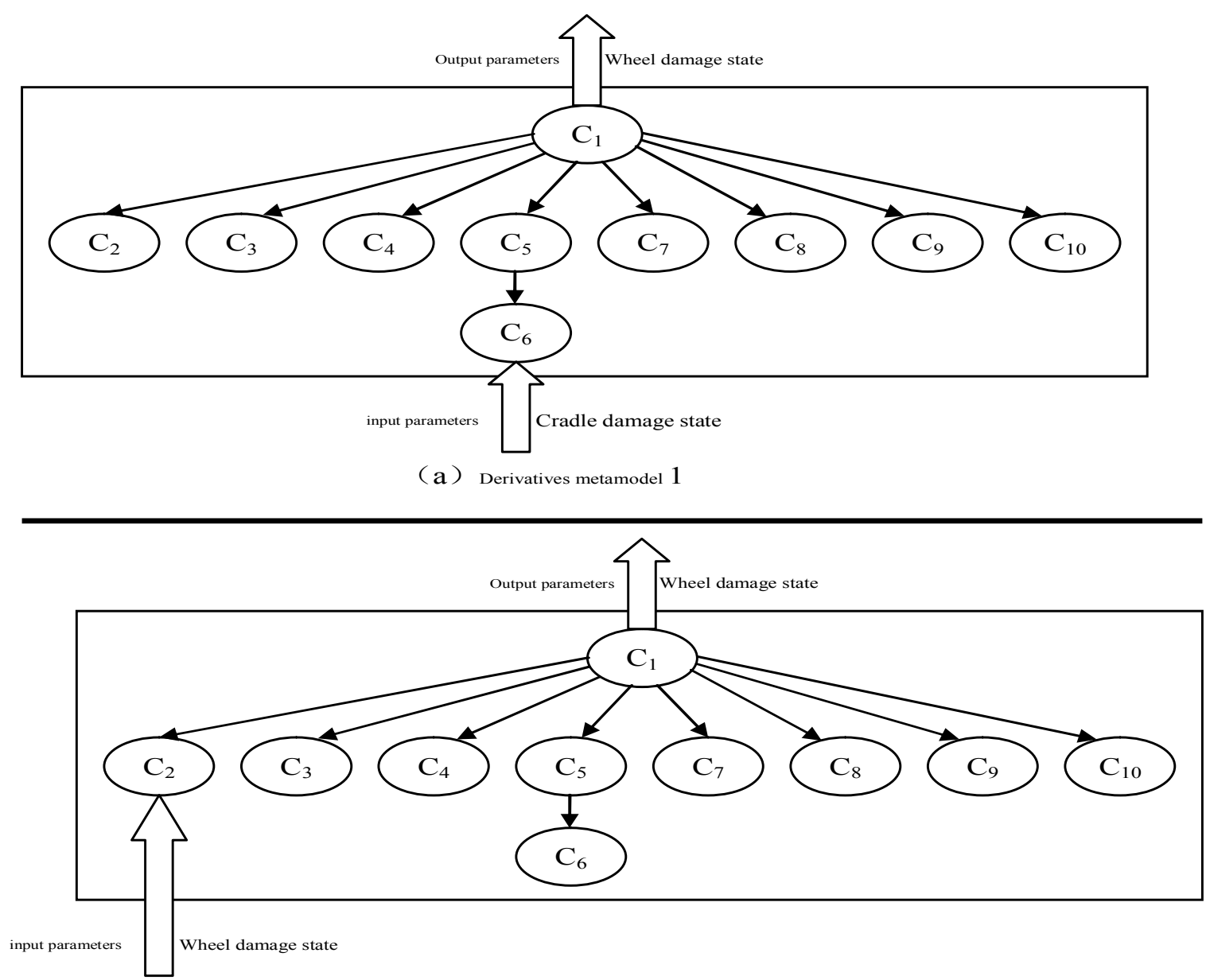

(b) Derivatives metamodel 2

Fig. 8 Schematic model derived element

Output of the model shown in Table 2, on the whole, in the case of proximity points, wheel damage state artillery leg injuries for increased sensitivity to changes in the state, that is, with increasing state Antiaircraft leg injury, damage occurs state 4 wheels probability increases. Derived metamodel 2 Antiaircraft leg injury prone as input parameters, in order to calculate the output parameter wheel damage state. According to the sensitivity of the change in the output parameters, the injury-prone parts of the meta-model input parameters are more reasonable

\section{Conclusions}

Using equipment battlefield damage simulation system to study the Bayesian network simulation metamodel. When Bayesian network topology is a key factor affecting the build quality of the Bayesian network meta-model simulation, using the $\mathrm{K} 2$ algorithm to find the optimal Bayesian network structure, it should be reasonably determined variable ordering, the proposed approach based methods to determine the energy transfer battlefield Damage applies correlation analysis to build a Bayesian network simulation metamodel.

Of course, it can also directly determine the corresponding Bayesian network meta-model simulation model based on the original work of the internal mechanisms, such as the literature[7].

\section{References}

[1]LI Jian-ping, WANG Wei-ping, BAI Feng-shan, ZHANG Jun.Metamodel Method FOR Two-level Optimality Analysis of Resource Allocating Problem[J]. Journal of System Simulation. 2008.20(4):837-840. 
[2]Russell R. Barton. Simulation metamodels. Proceedings of the 1998 Winter Simulation Conference. 1998.

[3]Robert G. Sargent. Research issues in metamodeling[C]. Proceedings of the 1991 Winter Simulation Conference. 1991.

[4]Zhang Lian-Wen, Guo Hai-Peng. Introduction to Bayesian Network [M]. Science Press. 2006:33-34.

[5]Haikuan-Wang, Quan Shi, Fei Xiong. The Application of TOPICS on Sequencing Decision-Making in Equipment Battlefield Repair. QR2MSE. 2013,1624-1628.

[6]ZHAO Bing, WANG Hai-kuan, SHI Quan. The Grade Model of Battlefield Damage Assessment for Equipment based on Weight of Classification of Index \& Unasertained Measure. MATHEMATICS IN PRACTICE AND THEORY. 2014.44(4):1351-1356.

[7]WANG Guang-yan, MA Zhi-jun, HU Qi-wei.The Fault Tree Analsis Based on Bayesian Networks.Systems Engineering-theory \& Practice.2004.24(6):78-83. 